\title{
Stochastic Geometry based Jamming Games in Mobile Ad hoc Networks
}

\author{
Manjesh K. Hanawal ${ }^{\dagger *}$ and Eitan Altman ${ }^{\dagger}$ \\ $\dagger$ INRIA B.P.93, 2004 Route des Lucioles 06902 Sophia-Antipolis Cedex, FRANCE \\ * LIA, University of Avignon, 339, chemin des Meinajaries, Avignon, FRANCE
}

\begin{abstract}
This paper studies the performance of a Poisson Mobile Ad hoc NETwork (MANET), that is owned by an Operator, in the presence of a Jammer. The objective of the Jammer is to degrade the spatial performance of the MANET by causing interference, whereas the Operator's objective is to set a Medium Access Probability (MAP) that optimizes it. The interaction between the Jammer and the Operator is modeled taking into account the costs related to transmission energy. This interaction is then transformed into a zero sum game by constructing an anti-potential. First, we consider the case where the receiver of a node is at a fixed distance and characterize the Nash equilibria by considering two spatial performance metrics: In the first case the Operator aims to maximize the number of successful transmissions per unit area, and in the second case the Operator aims to minimize the average delay per unit area. We then consider the case where distance between a transmitter and its receiver is not fixed. The Nash equilibria of the resulting game is again characterized.
\end{abstract}

Index Terms-Mobile Ad hoc Networks (MANET); Stochastic Geometry; zero-sum game;

\section{INTRODUCTION}

In this paper, we study an adversarial situation in wireless Mobile Ad hoc NETworks (MANETs) called the jamming game consisting of two players, namely, an Operator and a Jammer. The objective of the Operator is to maximize a suitably defined spatial performance metric and that of the Jammer is to minimize it. In addition, the players take into account the power consumption disutility incurred in order to achieve the throughput. The jamming game we consider amounts to choosing appropriate medium access probabilities in the presence of the adversary jammer. Viewing the channel and the available transmit power as resources, this jamming game can also be viewed as a resource allocation problem in the presence of an adversary.

Game theory is extensively used to study resource allocation problems in networks [15]. These games often include information theoretic aspects and/or communication theory. In that context, game theory has not only been applied to situations of competition over resources but also to adversarial situations, which can often be modeled as zero-sum games.

It is well known that computing the Nash equilibria in games is in general a hard problem. Indeed, this problem falls into a class of problems introduced by Christos Papadimitriou in 1994, called PPAD (Polynomial Parity Arguments on Directed graphs). In view of this complexity, it becomes attractive to identify classes of games for which one may compute the equilibria at a low complexity. We thus study a jamming game under some statistical assumptions on the mobility pattern, which are on one hand reasonable in many real scenarios, and on the other hand, allow for tractable and in several cases, even explicit expressions for the Nash equilibrium.

We consider slotted time, and assume that the mobiles are synchronized. The basic assumptions on our model are

- The location of the transmitters at each time slot forms a homogeneous Poisson point process.

- Mobility is high, so that the location of the mobiles at different slots is an independent and identically distributed (i.i.d) process.

- Medium access is controlled using Aloha

- Transmission success is based on SINR being larger than some threshold.

- Every mobile has always a packet to send.

The geometric aspects of the location of the nodes becomes important when the nodes are mobile; as the interference seen at a given location depends on the relative location of other nodes. In this paper we study a jamming problem in MANETs, in which the locations of the nodes form a homogeneous Poisson point process. We consider two models in Poisson MANETs introduced in [16]. First, we study Poisson bipolar model, in which the distance between the transmitter of a node and its receiver is fixed. We then consider a simple receiver selection model, where it is assumed that the set receivers form another Poisson point process that is independent of the transmitters.

Literature on Jamming Games: Jamming are among the first problems involving conflicts in networks that have been modeled and solved using game theory. The first publications on these games go thirty years back with the pioneering work [4], [5]. The question of the capacity achievable in channels prone to jamming was one of the main concerns, and was thus naturally studied within the information theory community, see e.g. [6], [7], [9]. For a recent survey on wireless games that includes jamming games, see [15]. Not only abstract jamming models have been studied using game theory, but also jamming of specific wireless local area networks, see [11] that study the jamming of IEEE 802.11. In some cases an adversary jammer may have access to signalling or information channels, and may be able to harm more by jamming these than by jamming the data transfer itself. Examples are jammers that interfere the signalling protocols, see e.g. [20] who study jamming signalling channels in a cellular network. [10] studies the jamming game in multihop radio networks with ALOHA multiple access scheme and Poisson assumption on the node distribution. The paper considers a scenario in which the network operator aims to maximize the expected forward 
progress of packets in a given direction, where as a Jammer aims to minimize it. The resulting Nash equilibria of the zero sum game are investigated numerically.

Our Contributions:The main contributions of the paper are the following:

- We model a jamming game in the Poisson MANETs with constraints on the transmission energies for both the Jammer and Operator. We introduce an anti-potential that allow us to the jamming game as a zero-sum game.

- Considering the Poisson bipolar model, we obtain explicit expression for the saddle points of the game by considering utilities based on throughput and delay. We then study the effect of the Jammer transmission power on the equilibrium. We shown that if the Operator chooses a transmit power higher than a certain level, then, at equilibrium, he/she can operate without the Jammer being active.

- In the receiver selection model, we again obtain explicit expressions for the saddle points considering throughput as the performance metric. We observe similar behavior as in the case of bipolar model.

The Poisson assumption on the location of nodes allows us to obtain utilities in a surprisingly simple explicit form, which in turn allows us to obtain much insight on the property of the equilibria and on the role of the transmission energy and its cost.

The Poisson assumption is valid when

- the number of mobiles in disjoint sets are independent;

- the number of mobiles in any given set follows a Poisson distribution. This class of point processes maximizes entropy. It is often used for modeling the location of users in e.g. mobile ad hoc networks.

In [21], in the context of cellular networks explicit expression are obtained for coverage and throughput with the Poisson assumption on the base stations. In [19], we study the Poisson MANET where each node is selfish. The Nash equilibria are characterized by considering the transmission energy costs.

The paper is organized as follows: Section II introduces the Poisson MANET model and discusses the performance metrics of interest. In Section III we model the jamming game and characterize the resulting equilibria of the Poisson bipolar model with density of successful transmission and density of delay as performance metrics. In Section IV we consider cases where the distance between a node's transmitter and its receiver is not fixed. Finally, we end with some concluding remarks in Section V.

\section{Model AND SETUP}

Consider the simplified mobile ad hoc network (MANET) model called the Poisson bipolar model proposed in [1]. Assume that each node follows the slotted version of the Aloha medium access control (MAC) protocol. Each dipole of the MANET consists of a transmitter and an associated receiver. We assume that each node has an infinite backlog of packets to transmit to its receiver. Nodes are scattered in the Euclidian space according to a homogeneous Poisson point process of intensity $\lambda_{1}$. Each node is associated with a multi- dimensional mark that carries information about the transmission status, fading condition, and the distance to receiver. We follow the notation of [3]. Let $\tilde{\Phi}=\left\{X_{i}, M_{i}\right\}_{i \geq 1}$ denote an independently marked Poisson point, where

- $\Phi=\left\{X_{i}\right\}_{i \geq 1}$ denotes the Poisson point process of intensity $\lambda_{1}$, representing the location of nodes in the Euclidean plane.

- $\left\{M_{i}=\left(S_{i}, e_{i}\right)\right\}_{i \geq 1}$ denotes the independent marks of the Poisson point process $\Phi$, which are made of three components:

- $S=\left\{S_{i}\right\}_{i \geq 1}$ denotes the channel condition between nodes and their associated receivers. It is assumed that channel conditions are independently and identically distributed (i.i.d.) across the nodes with a generic distribution denoted as $F$ with mean $1 / \mu$.

- $e=\left\{e_{i}\right\}_{i \geq 1}$ are indicator functions that take value 1 if a given node decides to transmit in a given time slot; otherwise they take value zero. They are assumed to be i.i.d. across the nodes.

- The processes $S, e$ are assumed to be independent of each other.

- We assume that distance between the transmitter and its associated receiver is at a fixed distance $r$.

Let $l(x, y)$ denote the attenuation function between any two given points $x, y \in \mathbb{R}^{2}$. We assume that this function just depends on the distance between points, i.e., $|x-y|$. With a slight abuse of notation we denote this function as $l(x, y)=$ $l(|x-y|)$. We assume the following form for this attenuation function

$$
l(x, y)=(A|x-y|)^{-\beta} \text { for } A>0 \text { and } \beta>2 .
$$

The marks $\left\{e_{i}\right\}_{i \geq 1}$ indicate if a given node transmits in a given time slot. Then the probability that the $i$ th node transmits is $q_{1}:=\operatorname{Pr}\left\{e_{i}=1\right\}=\mathbb{E}[e]$ (Medium Access Probability (MAP)). Note that $\Phi$ defines a pair of independent Poisson process representing transmitters $\Phi^{1}=\left\{X_{i}, e_{i}=1\right\}$ and non transmitters $\Phi^{0}=\left\{X_{i}, e_{i}=0\right\}$ with intensities $q_{1} \lambda_{1}$ and $\left(1-q_{1}\right) \lambda_{1}$ respectively. We assume that the channel between the receiver of a given node and the transmitter of any other node is also distributed as the random variable $S$. All the nodes transmit at a fixed power denoted as $P_{1}$. Signal transmitted by a transmitter located at $X_{i}$ is successfully received at its associated receiver at location $y_{i}$ if the signal to interference and noise ratio (SINR) at location $y_{i}$ is larger than some threshold $T$, i.e.,

$$
S I N R_{i}:=\frac{P S l\left(\left|X_{i}-y_{i}\right|\right)}{I_{\tilde{\Phi}^{1}}+W}>T,
$$

where $W$ denotes the thermal noise power at the receiver and $I_{\tilde{\Phi}^{1}}$ denotes the shot noise of the Poisson point process $\Phi^{1}$, namely, $I_{\tilde{\Phi}^{1}}=\sum_{X_{i} \in \Phi^{1}} P_{1} S l\left(\left|X_{i}-y_{i}\right|\right)$.

Consider a typical node at the origin, $X_{0}=0$ with mark $M_{0}=\left(S_{0}, e_{0}\right)$. The typical node is said to be covered if (2) 
holds given that it is a transmitter, i.e, sends some packet. Then the coverage probability of the typical node is

$$
\mathbf{P}^{0}\left\{\frac{P S_{i} l(r)}{I_{\tilde{\Phi}^{1}}+W}>T \mid e_{0}=1\right\},
$$

where $\mathbf{P}^{0}$ denotes the Palm distribution [2][Chap. I] of the stationary marked Poisson point process $\tilde{\Phi}$. By using Slivnyak's theorem [2], the coverage probability of a typical node when all other nodes use MAP $p$ is evaluated in [1][3] and denoted as $p_{c}\left(r, p \lambda_{1}, T\right)$.

\section{A. Spatial Performance metrics}

Let us assume that all the nodes belong to a single Operator. If the nodes have information about other nodes in their vicinity, they can achieve a better performance by cooperating: Nodes moving from dense clusters to sparse clusters can increase their MAP as chances of collision reduce. Nodes moving from sparse clusters to dense clusters can reduce their MAP as chances of collision increase. We assume that, because of mobility, nodes cannot gather such information in each time slot and transmit with a MAP $q_{1}$ that is set by the Operator. Then the typical node is a transmitter with probability $q_{1}$ and a non-transmitter with probability $\left(1-q_{1}\right)$. We call the product of the MAP and the coverage probability of the typical node the goodput and denote it as

$$
g\left(q_{1}\right):=q_{1} p_{c}\left(r, q_{1} \lambda_{1}, T\right)
$$

The set of nodes that transmit in a given slot form another Poisson point process of intensity $\lambda_{1} q_{1}$. Let $d_{s}\left(q_{1}\right)$ denote the average number of successful transmissions in a unit area. By Campbell's formula [2][Sec. 2.1.2] for stationary Poisson point processes this spatial performance metric is given as the product of goodput and the intensity of the Poisson point process, i.e., $d_{s}\left(q_{1}\right)=\lambda_{1} q_{1} p_{c}\left(r, q_{1} \lambda_{1}, T\right)$. We shall also consider the mean delay experienced by the nodes in successfully transmitting the packets to their associated receiver. We assume that if a packet transmission fails then the packet is retransmitted till success. We also assume that nodes can resample the channel in each slot. Recall our assumption on the mobility model that locations of the nodes are i.i.d across the time slot. Then by Little's theorem [12] the mean delay for a typical node is given by reciprocal of its goodput given in (4). Let $d_{t}\left(q_{1}\right)$ denote the spatial mean delay of nodes per unit area. Then again using Campbell's formula we get $d_{t}\left(q_{1}\right)=\lambda_{1} / q_{1} p_{c}\left(r, q_{1} \lambda_{1}, T\right)$. We refer to this quantity as spatial density of delay. Note that we used the i.i.d assumption on the nodes realization only to derive the spatial mean delay, and not on the performance metric goodput.

In the following sections we analyze the spatial performance of the network in the presence of a Jammer. In the next section we introduce our game model. We then analyze the jamming game considering the Poisson bipolar model with density of success and density of delay as spatial performance metrics.

\section{JAMMING GAME}

In this section we consider a jamming game in the Poisson bipolar MANET. Assume that there is a Jammer who also has nodes that are scattered according to a Poisson point process in the same geographical area. The Jammer aims to degrade the performance of the Operator by causing interference.

Let $\lambda_{2}$ denote the intensity of the nodes of the Jammer. The transmitters of the Jammer can resample the channel in each time slot and transmit at a fixed power $P_{2}$. If a transmitter of the Jammer is surrounded by a dense cluster of nodes belonging to the Operator, then the degradation in performance due to the interference from the Jammer may not be significant; as the success probability is already low. On the contrary, if a transmitter of the Jammer is surrounded by a sparse cluster of nodes belonging to the Operator then, the degradation in performance due to interference from the Jammer could be significant. However, we assume that, due to random mobility, the Jammer cannot gather such information. Also the Jammer incurs costs from the energy transmissions. Thus we assume that the Jammer keeps each of its transmitters turned ON with probability $q_{2}$ independently of its other transmitters. The transmitters of the Jammer form a Poisson point process of intensity $q_{2} \lambda_{2}$.

Recall that $q_{1}$ denotes the MAP set by the Operator. A transmission of a typical node of the Operator gets interference from all the other nodes that are transmitting. Thus a typical node gets interference from the nodes that form a Poisson point process of intensity $q_{1} \lambda_{1}+q_{2} \lambda_{2}$. In this section we assume that the channel between the receiver of the typical node and transmitters of all other nodes, both the Jammer and the Operator, is distributed according to the random variable $S$ and take the same path loss model in (1). The probability that a transmissions by the typical node is successful in the presence of the Jammer can be easily computed following the same steps as in [1][Lemma 3.2]. With some abuse of notation we represent this quantity by $p_{c}\left(q_{1}, q_{2}\right)$. The proof of the following lemma is given in Appendix B.

Lemma 1: Let each node of the Operator in the Poisson bipolar MANET transmit with MAP $q_{1}$ and let the Jammer turns ON each of its transmitters with probability $q_{2}$. Assume that $S$ is Rayleigh distributed with parameter $\mu$ and noise power is zero, then

$$
p_{c}\left(q_{1}, q_{2}\right)=\exp \left\{-C\left(q_{1} \lambda_{1}+q_{2} \lambda_{2} Q\right)\right\},
$$

where $C=2 \pi r^{2} T^{2 / \beta} K(\beta)$, and $Q=\left(P_{2} / P_{1}\right)^{2 / \beta}$

In the following, we consider performance metrics that are a monotonically increasing functions of this success probability. Let $d\left(q_{1}, q_{2}\right)$ denote the generic performance metric of the Poisson MANET that the Operator is interested in. In the remaining of the paper when we write a performance metric with two arguments $q_{1}$ and $q_{2}$, it is understood that the it is calculated in the presence of the Jammer who turns ON each of its node with probability $q_{2}$, and the nodes of the Operator uses MAP $q_{1}$.

From Equation (5) it is clear that the Operator can improve the performance of its nodes by increasing their transmission 
power, whereas the jammer can decrease it by increasing the transmitting power of its nodes. Thus it is beneficial for both the Operator and the Jammer to increase the transmission power of their nodes. However, we assume that each node incurs a transmission cost due to limited power available to them. The average density of power dissipated among the nodes of the Operator is $\lambda_{1} q_{1} P_{1}$ and that among the nodes of the Jammer is $\lambda_{2} q_{2} P_{2}$. For jamming games with energy constraints in cellular networks see [18]. Let $\rho_{1}$ and $\rho_{2}$ denote the cost incurred by the Operator and the Jammer per unit power transmitted, respectively. More formally, we define the strategy, utility, and objective of the game between the Jammer and the Operator as following:

Strategy: The strategy of the Operator is to choose a MAP $q_{1} \in[0,1]$ with which each of its nodes can access the channel. The strategy of the Jammer is to set a probability $q_{2} \in[0,1]$ with which a transmitter is turned $\mathrm{ON}$ in a given slot. Denote the joint strategy as $\mathbf{q} \in\left(q_{1}, q_{2}\right)=[0,1] \times[0,1]$.

Utilities and objective: The objective of the Operator is to choose a MAP $q_{1}$ that maximizes the density of success of its nodes taking into account average transmission costs among its nodes. We define the utility of the Operator as

$$
U_{1}\left(q_{1}, q_{2}\right)=d\left(q_{1}, q_{2}\right)-\rho_{1} \lambda_{1} q_{1} P_{1} .
$$

The objective of the Jammer is to set a probability $q_{2}$, with which each of its transmitters is turned $\mathrm{ON}$ in a given slot, that minimizes the density of the successful transmission of the Operator taking into account the average transmission costs incurred among its transmitters. We define the utility of the Jammer as

$$
U_{2}\left(q_{1}, q_{2}\right)=-d\left(q_{1}, q_{2}\right)-\rho_{2} \lambda_{2} q_{2} P_{2} .
$$

In terms of the optimization problems, we can write the objective of the Operator as to choose a $q_{1}^{*}$ such that

$$
q_{1}^{*} \in \operatorname{argmax}_{q_{1} \in[0,1]} U_{1}\left(q_{1}, q_{2}\right),
$$

and for the Jammer it is to choose a $q_{2}^{*}$ such that

$$
q_{2}^{*} \in \operatorname{argmax}_{q_{2} \in[0,1]} U_{2}\left(q_{1}, q_{2}\right) .
$$

Note that the jamming game with the utilities in (6) and (7) is not a zero sum game. This game can be studied as a zero sum game with an anti- potential function obtained by modifying the utilities of the players as below.

Anti potential: If we add the term $\rho_{2} \lambda_{2} q_{2} P_{2}$ to the utility function of the Operator, then the set of optimal values $q_{1}^{*}$ in equation (8) does not change. We define the modified utility function of the Operator as

$$
\left.\bar{U}_{1}\left(q_{1}, q_{2}\right)=d_{(} q_{1}, q_{2}\right)-\rho_{1} \lambda_{1} q_{1} P_{1}+\rho_{2} \lambda_{2} q_{2} P_{2}
$$

Similarly, if we add the term $\rho_{1} \lambda_{1} q_{1} P_{1}$ to the utility function of the Jammer, then the set of optimal values $q_{2}^{*}$ in equation (9) does not change. We define the modified utility function of the Jammer as

$$
\bar{U}_{2}\left(q_{1}, q_{2}\right)=-d\left(q_{1}, q_{2}\right)-\rho_{2} \lambda_{2} q_{2} P_{2}+\rho_{1} \lambda_{1} q_{1} P_{1} .
$$

If we consider a new game with this modified utilities, then the resulting equilibrium, if exits, will be same as in the original game. Notice that the modified utilities are such that $\bar{U}_{1}(\mathbf{q})=$ $-\bar{U}_{2}(\mathbf{q})$ for all $\mathbf{q}$. Hence, the new game constitutes a zero sum game. In the following subsections we consider performance metrics discussed in Subsection II-A, and analyze the resulting equilibria.

\section{A. Spatial density of Success as performance metric}

In this subsection we assume that the Operator is interested in the number of successful transmission per unit area. We refer to this performance metric as density of successful transmissions, and can be computed as

$$
d\left(q_{1}, q_{2}\right)=\lambda_{1} q_{1} p_{s}\left(q_{1}, q_{2}\right) .
$$

The anti-potential of the jamming game with this performance metric is given by

$$
\begin{array}{r}
\bar{U}_{1}\left(q_{1}, q_{2}\right)=\lambda_{1} q_{1} \exp \left\{-C\left(q_{1} \lambda_{1}+q_{2} \lambda_{2} Q\right)\right\} \\
-\lambda_{1} q_{1} \rho_{1} P_{1}+\lambda_{2} q_{2} \rho_{2} P_{2} .
\end{array}
$$

The following lemma states relevant properties of this antipotential function $\bar{U}_{1}(\mathbf{q})$.

Lemma 2: $\bar{U}_{1}\left(q_{1}, q_{2}\right)$ is a quasi concave function in $q_{1}$ and convex in $q_{2}$. Assume that $\rho_{1} P_{1}<\exp \left\{-\lambda_{2} C Q\right\}$, then $\bar{U}_{1}\left(\cdot, q_{2}\right)$ achieves the maximum in the interval $(0,1]$ if $\lambda_{1} C \geq 1$.

Proof: See Appendix in C.

The utility function $\bar{U}_{1}\left(q_{1}, q_{2}\right)$ is continuous in both the arguments. The strategy space of both the players is convex and compact. From Lemma 2, and using the Sion's minmax theorem [22] we can readily conclude that the jamming game has a saddle point, i.e., there exists a $\mathbf{q}^{*}=\left(q_{1}^{*}, q_{2}^{*}\right)$ such that for all $\left(q_{1}, q_{2}\right)$

$$
\bar{U}_{1}\left(q_{1}, q_{2}^{*}\right) \leq \bar{U}_{1}\left(q_{1}^{*}, q_{2}^{*}\right) \leq \bar{U}_{1}\left(q_{1}^{*}, q_{2}\right) .
$$

By transforming the jamming game into a zero-sum game we easily concluded that the saddle point of the jamming game exists. We can also explicitly obtain the saddle point as given in the following proposition. The saddle points are characterized in terms of the Lambert function [14] which we denote as $W(x)$.

Proposition 1: Assume that $\lambda_{1} C \geq 1$. The Nash equilibrium of the jamming game are as following. If $\rho_{1} P_{1} \geq 1$, then $(0,0)$ is an equilibrium. If $\rho_{1} P_{1}<\exp \left\{-\lambda_{2} C Q\right\}$ we have the following cases.

- If $\rho_{2} P_{2} \geq \tilde{q}_{1} \lambda_{1} C Q \exp \left\{-\tilde{q}_{1} \lambda_{1} C\right\}$ then $\left(\tilde{q}_{1}, 0\right)$ is an equilibrium, where $\tilde{q}_{1}$ is given by

$$
\tilde{q}_{1}=\frac{1-W\left(e \rho_{1} P_{1}\right)}{\lambda_{1} C} .
$$

- If $\rho_{2} P_{2} \leq \bar{q}_{1} \lambda_{1} C Q \exp \left\{-\bar{q}_{1} \lambda_{1} C\right\} \exp \left\{-C \lambda_{2} Q\right\}$ then $\left(\bar{q}_{1}, 1\right)$ is an equilibrium, where $\bar{q}_{1}$ is given by

$$
\bar{q}_{1}=\frac{1-W\left(e \rho_{1} P_{1} \exp \left\{\lambda_{2} C Q\right\}\right)}{\lambda_{1} C} .
$$


If $\left(q_{1}^{*}, q_{2}^{*}\right)$ is an interior equilibrium point then

$$
q_{1}^{*}=\frac{1}{\lambda_{1} C\left(1+Q\left(\rho_{1} P_{1} / \rho_{2} P_{2}\right)\right)},
$$

and

$$
q_{2}^{*}=\frac{-1}{C Q} \log \left\{\frac{\rho_{2} P_{2} \exp \left\{\lambda_{1} q_{1}^{*} C\right\}}{\lambda_{1} q_{1}^{*} C Q}\right\} .
$$

Proof: See Appendix A

One can interpret the results of the above proposition as follows. Under the condition $\rho_{1} P_{1} \geq 1$ the utility of the Operator is always negative, i.e., the total transmission energy cost for the Operator is high, hence it is optimal for the Operator not to allow any transmissions from its nodes. Once the nodes of the MANET are not active then the Jammer will also turn OFF its transmitter. The condition in the first bullet give a scenario in which the operating cost for the Jammer is too high to allow any transmissions from its nodes. Hence the Jammer keeps all of its nodes turned OFF at equilibrium. The condition in the second bullet gives a scenario in which the transmission costs for the Jammer is low and all the transmitters are turned ON at equilibrium. Equation (13) results in an interior point if

$$
P_{2} \rho_{2}<q_{1}^{*} \lambda_{1} Q C \exp \left\{-q_{1}^{*} \lambda_{1} C\right\}
$$

and

$$
P_{2} \rho_{2}>Q C q_{1}^{*} \lambda_{1} \exp \left\{-q_{1}^{*} \lambda_{1} C\right\} \exp \left\{-\lambda_{1} C Q\right\}
$$

where $q_{1}^{*}$ is given by Equation (12).

Let us proceed to study the effect of power and cost factors on the equilibria. Let $q_{1}^{*}\left(q_{2}\right)$ denote the best response of the Operator when the Jammers' strategy is $q_{2}$. From Equation (37) it is easy to note that the best response of the Operator is a decreasing function in $q_{2}$. Hence $\bar{q}_{1} \leq q_{1}^{*}\left(q_{2}\right) \leq \tilde{q}_{1}$ for all $q_{2} \in\left(\begin{array}{ll}0 & 1\end{array}\right)$. Where $\tilde{q}_{1}$ is the best response when $q_{2}=$ 0 , and $\bar{q}_{1}$ is the best response when $q_{2}=1$. To understand the bounds on the product $\rho_{2} P_{2}$ given in Proposition 1 more clearly, let us define a function $f:[0,1] \rightarrow \mathbb{R}_{+}$given by $f(q)=q \lambda_{1} C \exp \left\{-q \lambda_{1} C\right\}$. The function $f$ attains maxima at $q=1 / \lambda_{1} C$, and is increasing in the interval $\left[0,1 / \lambda_{1} C\right]$. Thus it is clear that

$$
f\left(\tilde{q}_{1}\right) \geq f\left(q_{1}^{*}\left(q_{2}\right)\right) \geq f\left(\bar{q}_{1}\right) \text { for all } q_{2} \in\left(\begin{array}{ll}
0 & 1
\end{array}\right) .
$$

This verifies the consistency of the bounds on $P_{2} \rho_{2}$ given in Proposition 1 and are shown in Figure 1.

Note that the upper bound and lower bound on $\rho_{2} P_{2}$ in Proposition 1 that determines the value of equilibrium $\left(q_{1}, q_{2}\right)$ are functions of the power transmitted by the Jammer and the operator. In Figure 2, we show the variations of $Q f\left(\bar{q}_{1}\right) \exp \left\{-\lambda_{2} C Q\right\}$ and $Q f\left(\tilde{q}_{1}\right)$ as a function of $P_{2}$ for a given value of system parameters, price factors, and the power $P_{1}$ transmitted by the nodes of the Operator. In the region marked $A, P_{2}$ satisfies the relation

$$
\rho_{2} P_{2} \leq Q C \tilde{q}_{1} \lambda_{1} \exp \left\{-\tilde{q}_{1} \lambda_{1} C\right\} \exp \left\{-\lambda_{1} C Q\right\}
$$

If the power transmitted by the Jammer lies in this region, then the Jammer turns $\mathrm{ON}$ all of his nodes at equilibrium. In the region marked $C, P_{2}$ satisfies the relation

$$
P_{2} \rho_{2} \geq \bar{q}_{1} \lambda_{1} Q C \exp \left\{-\bar{q}_{1} \lambda_{1} C\right\}=Q f\left(\bar{q}_{1}\right) .
$$

If the power transmitted by the Jammer lies in this region, then, at equilibrium, all the nodes of the Jammer are turned OFF. In the region marked $B, P_{2}$ satisfies the relation

$$
P_{2} \rho_{2}<\bar{q}_{1} \lambda_{1} Q C \exp \left\{-\bar{q}_{1} \lambda_{1} C\right\}
$$

and

$$
P_{2} \rho_{2}>Q C \tilde{q}_{1} \lambda_{1} \exp \left\{-\tilde{q}_{1} \lambda_{1} C\right\} \exp \left\{-\lambda_{1} C Q\right\} .
$$

If the power transmitted by the Jammer lies this region $B$, then the Jammer turns ON each of its nodes with probability that is an interior point at equilibrium.

In Figure 3, we plot the best response (BR) of the Jammer and the Operator. We choose one power level, $P_{2}$, for the Jammer from each of the region $A, B$, and $C$, with the power level for the Operator fixed at the same value as that used in Figure 2. In Figure 3 the blue lines correspond to the best response of the Operator, and the red lines correspond to that of the Jammer. The equilibrium for the corresponding value of $P_{2}$ are marked.

Nullifying the Jamming effect:For a given power level and the cost factor of the Jammer if the condition $P_{2} \rho_{2} \geq Q f\left(\tilde{q}_{1}\right)$ holds at equilibrium, then the Operator sees no Jamming effect as all the nodes of the Jammer are turned OFF. Thus it is interesting for the Operator to choose a power level such that the Jammer turns OFF its nodes at equilibrium. It is easy to note the $Q f\left(\tilde{q}_{1}\right)$ is a decreasing function in $P_{1}$. Indeed, $f\left(\tilde{q}_{1}\right)$ is a decreasing function in $P_{1}$ as $\tilde{q}_{1}$ is less than $\leq 1 / \lambda_{1} C$ and decreasing in $P_{1}$ (see Equation (10)). Hence the product $Q f\left(\tilde{q}_{1}\right)$ is also decreasing in $P_{1}$. Thus the Operator can increase his power level, say to $P_{1}^{*}$, such that $P_{2} \rho_{2} \geq Q f\left(\tilde{q}_{1}\right)$, and operate without the Jamming effect at equilibrium. However, the Operator can do so provided its cost factor is such that $\rho_{1} P_{1}^{*} \leq 1$.

\section{B. Spatial density of delay as performance metric}

In this section we assume that that the Operator aims to minimize the spatial density of delay, whereas the Jammer aims to maximize it. From the arguments in Subsection II-A, we can write the spatial density of delay as the reciprocal of the spatial density of success. Continuing the set up used in the jamming game model, we write the performance metric and the resulting anti-potential as

$$
d\left(q_{1}, q_{2}\right)=-\frac{\lambda_{1} \exp \left\{C\left(\lambda_{1} q_{1}+\lambda_{2} q_{2} Q\right)\right\}}{q_{1}}
$$

and

$$
\begin{array}{r}
\bar{U}_{1}\left(q_{1}, q_{2}\right)=-\frac{\lambda_{1} \exp \left\{C\left(\lambda_{1} q_{1}+\lambda_{2} q_{2} Q\right)\right\}}{q_{1}} \\
-\rho_{1} \lambda_{1} q_{1} P_{1}+\rho_{2} \lambda_{2} q_{2} P_{2}
\end{array}
$$




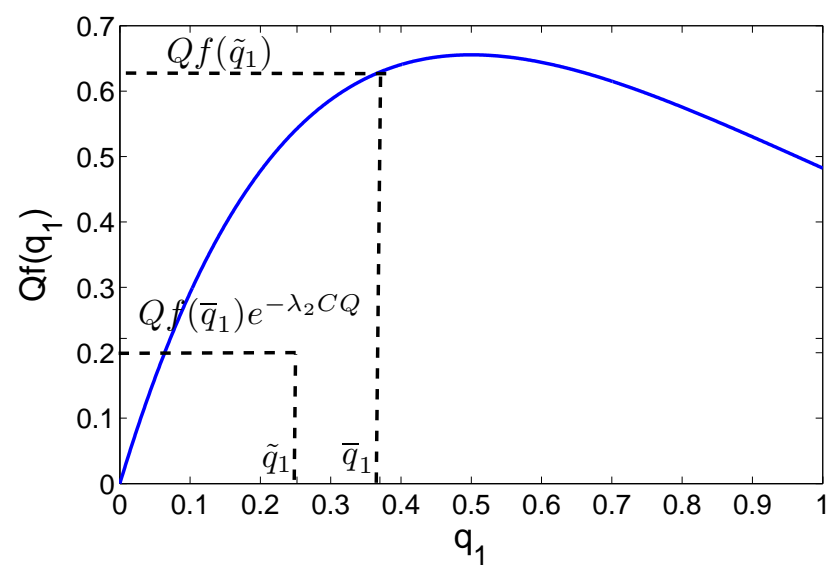

Figure 1. Price Bounds: $\lambda_{1}=2, \lambda_{2}=.5, C=1, \rho_{2}=.8, \rho_{1}=$ $0.41, P 1=0.3, P_{2}=0.6, \beta=2.4$

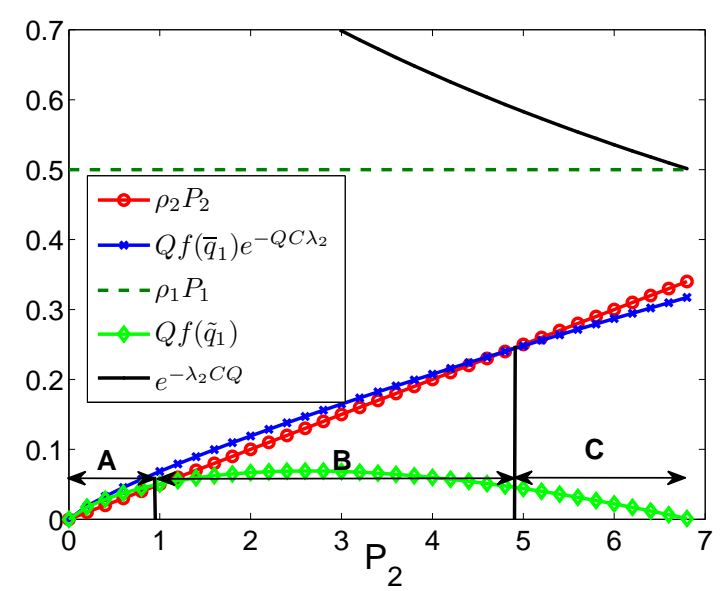

Figure 2. Price Region: $\lambda_{1}=2, \lambda_{2}=.5, \rho=.11, \rho_{2}=0.05, \beta=$ $2.5, P_{1}=4.54$

The following lemma states the properties of this anti-potential function.

Lemma 3: $\bar{U}_{1}\left(q_{1}, \cdot\right)$ is a concave function for all $q_{1} \in$ $[0,1] . \bar{U}_{1}\left(\cdot, q_{2}\right)$ is a concave function for all $q_{2} \in[0,1]$, and attains maximum at an argument within the interval $q_{1} \in[0,1]$ if $\lambda_{1} C \geq 1$.

Proof: See Appendix in D.

From the above lemma we see that the hypothesis of Sion's minmax theorem [22] holds. Hence the Nash equilibrium exists. The following proposition characterizes the Nash equilibria.

Proposition 2: Assume that $\lambda_{1} C \geq 1$.

- Let $\bar{q}_{1}$ be the solution of

$$
\frac{\lambda_{1}}{\left(\bar{q}_{1}\right)^{2}} \exp \left\{\left(\lambda_{1} \bar{q}_{1}+\lambda_{2} Q\right)\right\}\left(1-C \lambda_{1} \bar{q}_{1}\right)=\rho_{1} P_{1}
$$

and $\left(\lambda_{2} / \lambda_{1}\right) \rho_{2} P_{2} \bar{q}_{1} \exp \left\{-\lambda_{1} C \bar{q}_{1}\right\}<\exp \left\{\lambda_{2} C Q\right\}-1$. Then $\left(\bar{q}_{1}, 1\right)$ is the Nash equilibrium.

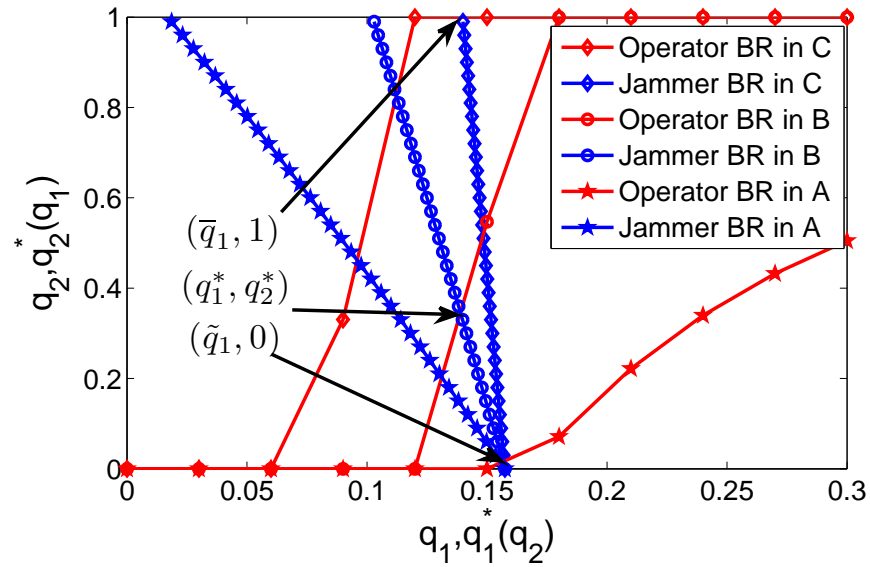

Figure 3. Best Response: $\lambda_{1}=2, \lambda_{2}=.5, \rho=.11, \rho_{2}=0.05, \beta=$ $2.5, P_{1}=4.54, P_{2}=0.5$

- Let $\tilde{q}_{1}$ be the solution of

$$
\frac{\lambda_{1}}{\left(\tilde{q}_{1}\right)^{2}} \exp \left\{\lambda_{1} C \tilde{q}_{1}\right\}\left(1-C \lambda_{1} \tilde{q}_{1}\right)=\lambda_{1} \rho_{1} P_{1},
$$

and $\left(\lambda_{2} / \lambda_{1}\right) \rho_{2} P_{2} \tilde{q}_{1} \exp \left\{-\lambda_{1} C \tilde{q}_{1}\right\}>\exp \left\{\lambda_{2} C Q\right\}-1$. Then $\left(\tilde{q}_{1}, 0\right)$ is an equilibrium.

Proof: The proof follows by noting that $-\bar{U}_{1}\left(q_{1}, q_{2}\right)$ is convex in $q_{2}$ and hence maximized at the extreme points.

\section{Receiver Selection Models}

In the Poisson bipolar model of the last section we assumed that the receiver of a node is at a fixed distance. In this section we relax this condition. We consider two models for receiver selection introduced in [16][Ch 17], namely, independent nearest receiver (INR) model and MANET nearest receiver (MNR) model.

\section{A. Independent Nearest Receiver model}

In the INR model we assume that the potential receivers form a Poisson point process $\Phi_{0}$ of intensity $\lambda_{0}$ and is independent of the Poisson point process $\Phi$. Each active node of the Operator aims to transmit to the receiver that is nearest to it. Let the random variable $R$ denote the distance between a typical node at the origin and to its nearest receiver. The probability density function of $R$, denoted as $F_{R}$, is given by

$$
F_{R}(r)=2 \pi \lambda_{0} r \exp \left\{-\lambda_{0} \pi r^{2}\right\}, \quad r \geq 0 .
$$

Now consider the jamming game of Section III. Recall that the Jammer turns $\mathrm{ON}$ each of its nodes with probability $q_{2}$ and uses the power $P_{2}$ to cause interference to the nodes of the Operator. Let $d_{s}\left(\mathrm{INR}, q_{1}, q_{2}\right)$ denote the density of the successful transmissions of the Operator. Following the steps of Lemma 1 and Proposition 17.2 in [16], we have

$$
\begin{aligned}
d_{s} & \left(\mathrm{INR}, q_{1}, q_{2}\right) \\
& =\lambda_{1} q_{1} \int_{r \geq 0} r \exp \left\{-\pi r^{2} C_{1}\left(q_{1} \lambda_{1}+q_{2} \lambda_{2} Q\right)\right\} \mathrm{dF}_{\mathrm{R}}(\mathrm{r}) \\
& =\frac{\lambda_{1} \lambda_{0} q_{1}}{C_{1}\left(q_{1} \lambda_{1}+q_{2} \lambda_{2} Q\right)+\lambda_{0}}
\end{aligned}
$$


where $C_{1}=2 T^{2 / \beta} K(\beta)$. Let $\rho_{1}$ and $\rho_{2}$ denote the cost factor of the Operator and Jammer respectively. We define the utility of the Operator and the Jammer with the energy cost as

$$
\begin{aligned}
& U_{1}\left(\mathrm{INR}, q_{1}, q_{2}\right) \\
& =d_{s}\left(\mathrm{INR}, q_{1}, q_{2}\right)-\lambda_{1} q_{1} \rho_{1} P_{1} \\
& =\frac{\lambda_{1} \lambda_{0}\left(1-\rho_{1} P_{1}\right)-\lambda_{1} \rho_{1} P_{1} C_{1}\left(q_{1} \lambda_{1}+q_{2} \lambda_{2} Q\right)}{C_{1}\left(\lambda_{1}+q_{2} / q_{1} \lambda_{2} Q\right)+\lambda_{0} / q_{1}}
\end{aligned}
$$

and

$$
U_{2}\left(\mathrm{INR}, q_{1}, q_{2}\right)=-d_{s}\left(\mathrm{INR}, q_{1}, q_{2}\right)-\lambda_{2} q_{2} \rho_{2} P_{2},
$$

respectively. As in Section III, by adding $\lambda_{2} q_{2} \rho_{2} P_{2}$ to Equation (17) and $\lambda_{1} q_{1} \rho_{1} P_{1}$ to Equation (18), we have an antipotential function given by

$$
\begin{aligned}
& \bar{U}_{1}\left(\mathrm{INR}, q_{1}, q_{2}\right)=U_{1}\left(\mathrm{INR}, q_{1}, q_{2}\right)+\lambda_{2} q_{2} \rho_{2} P_{2}(19) \\
& \quad=-\left(U_{2}\left(\mathrm{INR}, q_{1}, q_{2}\right)+\lambda_{1} q_{1} \rho_{1} P_{1}\right)
\end{aligned}
$$

\section{B. MANET nearest receiver model}

In the MNR model we assume that each transmitter picks the nearest node of $\Phi$ that is not active in the considered time slot. Let the Operator choose the MAP $q_{1}$. Then the receivers form a Poisson point process $\Phi^{0}$ of intensity $\left(1-q_{1}\right) \lambda_{1}$ which is independent of the set of transmitters. Let $d_{s}\left(\mathrm{MNR}, q_{1}, q_{2}\right)$ denote the density of successful transmissions of the nodes of the Operator when the Jammer turns ON each of its nodes with probability $q_{2}$. We have [16][Eq. 17.6]

$$
d_{s}\left(\mathrm{MNR}, q_{1}, q_{2}\right)=d_{s}\left(\mathrm{INR}, q_{1}, q_{2}\right)
$$

with $\lambda_{0}=\left(1-q_{1}\right) \lambda$. We define the utility of the Operator with transmission cost as

$$
\begin{aligned}
& U_{1}\left(\mathrm{MNR}, q_{1}, q_{2}\right) \\
& =d_{s}\left(\mathrm{MNR}, q_{1}, q_{2}\right)-\lambda_{1} q_{1} \rho_{1} P_{1} \\
& =\frac{\lambda_{1}\left(1-q_{1}\right) \lambda_{1} q_{1}}{C_{1}\left(q_{1} \lambda_{1}+q_{2} \lambda_{2} Q\right)+\left(1-q_{1}\right) \lambda_{1}}-\lambda_{1} q_{1} \rho_{1} P_{1} \\
& =\frac{\lambda_{1}\left(1-\rho_{1} P_{1}\right)-q_{1} \lambda_{1}\left(\lambda_{1} \rho_{1} P_{1} C_{1}+\left(1-\lambda_{1} P_{1}\right)\right)}{C_{1}\left(1+q_{2} \lambda_{2} / q_{1} \lambda_{1} Q\right)+1 / q_{1}-1} \\
& \quad+\frac{\lambda_{1} \rho_{1} P_{1} C_{1} q_{2} \lambda_{2} Q}{C_{1}\left(1+q_{2} \lambda_{2} / q_{1} \lambda_{1} Q\right)+1 / q_{1}-1} .
\end{aligned}
$$

Comparing Equation (17) with Equation (21) we observe that both the utility functions have the same structure. Henceforth we restrict our analysis of the jamming problem to the case of INR model.

Saddle Point: It is clear that the anti-potential function $\bar{U}_{1}\left(\mathrm{INR}, q_{1}, q_{2}\right)$ is a concave function in the strategy of the Operator and a convex function in the strategy of the Jammer. Then we can directly apply Sion's minimax theorem to conclude that Saddle point of the Jamming game exists. The next proposition characterizes the equilibrium in the INR receiver selection model.

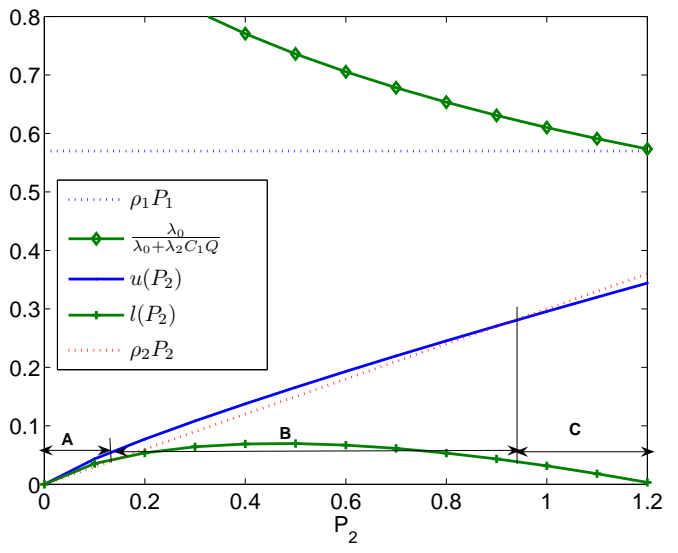

Figure 4. Price Region: $\lambda_{1}, \lambda_{2}, \lambda_{0}=1, C_{1}=.4, \rho_{1}=1, \rho_{2}=.3, \beta=$ $2.4, P_{1}=.57$

Proposition 3: The Nash equilibrium of the jamming game are as following. If $\rho_{1} P_{1} \geq 1$, then $(0,0)$ is an equilibrium. If $\rho_{1} P_{1}<\lambda_{0} /\left(C_{1} \lambda_{2} Q+\lambda_{0}\right)$ we have the following cases.

- If $\rho_{2} P_{2} \geq \frac{\lambda_{1} \lambda_{0} \tilde{q}_{1} C_{1} Q}{\left(C_{1} \tilde{q}_{1} \lambda_{1}+\lambda_{0}\right)^{2}}$ then $\left(\tilde{q}_{1}, 0\right)$ is an equilibrium, where $\tilde{q}_{1}$ is given by

$$
\tilde{q}_{1}=\min \left\{1, \frac{\left(\lambda_{0} / \sqrt{\rho_{1} P_{1}}-\lambda_{0}\right)}{\lambda_{1} C_{1}}\right\} .
$$

- If $\rho_{2} P_{2} \leq \frac{\lambda_{1} \lambda_{0} \bar{q}_{1} C_{1} Q}{\left(C_{1} \bar{q}_{1} \lambda_{1}+C_{1} \lambda_{2} Q \lambda_{0}+\lambda_{0}\right)^{2}}$ then $\left(\bar{q}_{1}, 1\right)$ is an equilibrium, where $\bar{q}_{1}$ is given by

$$
\bar{q}_{1}=\min \left\{1, \frac{\sqrt{\left(C_{1} \lambda_{2} Q \lambda_{0}+\lambda_{0}^{2}\right) / \rho_{1} P_{1}}-\lambda_{2} Q C_{1}-\lambda_{0}}{\lambda_{1} C_{1}}\right\}
$$

If $\left(q_{1}^{*}, q_{2}^{*}\right)$ is an interior equilibrium point, then

$$
q_{1}^{*}=\frac{\lambda_{0} Q \rho_{2} P_{2}}{\lambda_{1} C\left(\rho_{2} P_{2}+Q \rho_{1} P_{1}\right)^{2}}
$$

and

$$
q_{2}^{*}=\frac{1}{\lambda_{2} C_{1} Q}\left\{\sqrt{\left(\lambda_{0} \lambda_{1} q_{1}^{*} Q C_{1}\right) / \rho_{2} P_{2}}-C_{1} \lambda_{1} q_{1}^{*}-\lambda_{0}\right\} .
$$

Proof: See Appendix E.

Note that, unlike in Proposition 1 we did not put the restriction $\lambda_{1} C_{1} \geq 1$ in the above proposition. However, for Equation (24) and (25) to result in an interior point we need the conditions $\lambda_{0} Q \rho_{2} P_{2} \leq C_{1} \lambda_{1}\left(\rho_{2} P_{2}+Q \rho_{1} P_{1}\right)^{2}$, and

$$
\frac{\lambda_{1} \lambda_{2} \bar{q}_{1} C_{1} Q}{\left(C_{1} \bar{q}_{1} \lambda_{1}+C_{1} \lambda_{2} Q \lambda_{0}+\lambda_{0}\right)^{2}} \leq \rho_{2} P_{2} \leq \frac{\lambda_{1} \lambda_{2} \tilde{q}_{1} C_{1} Q}{\left(C_{1} \tilde{q}_{1} \lambda_{1}+\lambda_{0}\right)^{2}} .
$$

Figure 4 shows the bound on $\rho_{2} P_{2}$ as given in Proposition. This has similar shape as that in 2 . Hence, we can infer the same kind of results for the INR receiver selection model. 


\section{CONCLUSIONS}

Geometric considerations play a very central role in wireless communications, since the attenuation of wireless channels strongly depend on the distance between transmitter and receiver. Models that take into account the exact location of mobiles are often too complex to analyze or to optimize. Our objective in this paper is to analyze the performance of Poisson MANET in the presence of an adversarial Jammer and study the equilibrium properties.

More structured point processes can also be contemplated, for instance exhibiting attraction (hot spots) or repulsion (more elaborate medium access control than Aloha like e.g. CSMA). We leave the analysis of medium access games under such point processes for future research.

\section{REFERENCES}

[1] Baccelli, B. Blaszczyszyn and P. Múhlethaler, "An ALOHA protocol for multihop mobile wireless networks," IEEE Transactions on Information Theory, Vol 52, No. 2, pp. 421 - 436, Feb. 2006.

[2] F. Baccelli and B. Blaszczyszyn, "Stochastic Geometry and Wireless Networks Volume 1: THEORY,“ Foundations and Trends in Networking: Vol. 3: No 3-4, 2009, pp 249-449. http://dx.doi.org/10.1561/1300000006

[3] F. Baccelli, B. Blaszczyszyn, P. Múhlethaler, "Stochastic Analysis of Spatial and Opportunistic Aloha," IEEE journal on selected areas in communications, vol. 27, no. 7, sep 2009, pp 1105-1119

[4] T. Basar, "The Gaussian test channel with an intelligent jammer," IEEE Trans. Inform. Theory, vol. 29, pp. 152-157, Jan. 1983.

[5] T. Basar and Y.-W. WU, "A complete characterization of minimax and maximin encoder-decoder policies for communication channels with incomplete statistical description,” IEEE Trans. Inform. Theory, vol. 31, pp. 482-489, July 1985.

[6] M. Medard, "Capacity of correlated jamming channels," Allerton Annual Conf. on Comm., Control and Computing, 1997.

[7] M. H. Brady, M. Mohseni, and J. M. Cioffi,"Spatially-correlated jamming in Gaussian multiple access and broadcast channels," Proc. Confe. on Inform., Science, and Systems, Princeton, March 2006.

[8] S. Shafiee and S. Ulukus,"Capacity of multiple access channels with correlated jamming" Military Communications Conference, MILCOM, vol. 1, pp. 218-224, Oct. 2005.

[9] S. N. Diggavi and T. Cover, "The worst additive noise under a covariance constraint" IEEE Trans. Inform. Theory, vol. 47, pp. 3072-3081, Nov. 2001.

[10] J. Zander, "Jamming games in slotted Aloha packet radio network," IEEE Military Communications Conference, MILCOM '90, pp. 830 834 vol.2 30 sept. 1990 - 03 oct. 1990, Monterey, CA , USA

[11] E. Bayraktaroglu, C. Kingy, X. Liu, G. Noubir, R. Rajaraman, B. Thapa, "On the Performance of IEEE 802.11 under Jamming", Proceedings of Infocom 2008

[12] L. Kleinrock, "Queueing Systems. Volume 1: Theory," WileyInterscience; 1 edition

[13] S. Boyd, L. Vandenberghe, "Convex Optimization," Cambridge University Press 2004

[14] R. M. Corless, G. H. Gonnet, D. E. G. Hare, D. J. Jeffrey, D. E. Knuth, "On the Lambert W function," Advances in Computational Mathematics (Berlin, New York: Springer-Verlag) vol. 5, 1996, pp 329-359

[15] Mark Felegyhazi and Jean-Pierre Hubaux, "Game Theory in Wireless Networks: A Tutoria", EPFL Technical report: LCA-REPORT-2006-002.

[16] F. Baccelli and B. Blaszczyszyn, "Stochastic Geometry and Wireless Networks Volume 2: APPLICATIONS," Foundations and Trends in Networking: Vol. 4: No 1-2, 2009, pp 1-312. http://dx.doi.org/10.1561/1300000026

[17] Eitan Altman, Konstantin Avrachenkov and Andrey Garnaev, "Jamming in wireless networks: the case of several jammer", GameNets International Conference on Game Theory for Networks, 13-15 May 2009, Bogaziçi University, Istanbul, Turkey.

[18] Eitan Altman, Konstantin Avrachenkov, Andrey Garnaev, "A Jamming game in wireless networks with transmission cos", Net-COOP, Avignon, France, June 5-7, 2007. 2nd best paper Award.
[19] M.K.Hanawal, E. Altman, F. Baccelli, "Stochastic Geometric based Medium Access Games," Technical Report. Available at http://www-sop.inria.fr/members/Manjesh_Kumar.Hanawal/SG.pdf

[20] Saswati Sarkar, Eitan Altman, Rachid El-Azouzi and Yezekael Hayel, "Information Concealing games" Infocom symposium, 14 April 2008.

[21] Jeffrey G. Andrews, Francois Baccelli, R. K. Ganti, "A New Tractable Model for Cellular Coverage," 48th Allerton conference, Sep. 2010

[22] M. Sion, "On general minimax theorems", Pacific J. Math. Volume 8, Number 1 (1958), 171-176.

\section{APPENDIX A \\ PROOF OF PROPOSITION 1}

If $\rho_{1} P_{1} \geq 1$, then the slope of $\bar{U}\left(\cdot, q_{2}\right)$ is negative for all values of $q_{2}$. Thus $q_{1}=0$ is the unique maximizer of $U\left(0, q_{2}\right)$. If $q_{1}=0$ then $q_{2}=0$ is the unique minimizer of $\bar{U}\left(0, q_{2}\right)$. Hence $(0,0)$ is an equilibrium.

Differentiating $\bar{U}_{1}$ with respect to $q_{1}$ we have

$$
\begin{array}{r}
\frac{\partial \bar{U}\left(q_{1}, q_{2}\right)}{\partial q_{1}}=\lambda_{1} \exp \left\{-C\left(q_{1} \lambda_{1}+q_{2} \lambda_{2} Q\right)\right\} \\
\times\left(1-q_{1} \lambda_{1} C\right)-\lambda_{1} \rho_{1} P_{1}
\end{array}
$$

Assume that $\rho_{1} P_{1}<\exp \left\{-\lambda_{2} C Q\right\}$. Note that from Lemma $2, \bar{q}_{1}$ and $\tilde{q}_{1}$ lies in the interval $[0,1]$. Let $q_{2}=0$, then it follows from Equation (37) and the definition of the Lambert function [14], that the maximizer of $\bar{U}_{1}(\cdot, 0)$ is $(10)$. Let $q_{1}=$ $\tilde{q}_{1}$, the slope of $\bar{U}_{1}\left(\tilde{q}_{1}, \cdot\right)$ is

$$
\begin{aligned}
& \partial \bar{U}_{1}\left(\tilde{q}_{1}, q_{2}\right) / \partial q_{2}= \\
& \quad-\quad q_{1} \lambda_{1} C Q \exp \left\{-\tilde{q}_{1} \lambda_{1} C\right\} \exp \left\{-C Q q_{2} \lambda_{2}\right\}+\rho_{2} P_{2},
\end{aligned}
$$

which is positive for all values of $q_{2}$ under the hypothesis of the first bullet. This implies that $\bar{U}_{1}\left(\tilde{q}_{1}, \cdot\right)$ is an increasing function in $q_{2}$. Then $q_{2}=0$ is the optimal strategy for the Jammer. This verifies the claim in the first bullet. Assume $q_{2}=1$, then it follows from Equation (37) and the definition of the Lambert function, that the maximizer of $\bar{U}_{1}(\cdot, 1)$ is $(11)$. Let $q_{1}=\bar{q}_{1}$, the slope of $\bar{U}_{1}\left(\bar{q}_{1}, \cdot\right)$ is

$$
\begin{aligned}
& \partial \bar{U}_{1}\left(\bar{q}_{1}, q_{2}\right) / \partial q_{2}= \\
& \quad-\quad q_{1} \lambda_{1} C Q \exp \left\{-\bar{q}_{1} \lambda_{1} C\right\} \exp \left\{-C Q q_{2} \lambda_{2}\right\}+\rho_{2} P_{2},
\end{aligned}
$$

which is negative for all values of $q_{2}$ under the hypothesis of the second bullet. This implies that $\bar{U}_{1}\left(\bar{q}_{1}, \cdot\right)$ is a decreasing function in $q_{2}$. Then the optimal value for the Jammer is to set $q_{2}=1$. This verifies the claim in second bullet. Let $\left(q_{1}^{*}, q_{2}^{*}\right)$ be an interior equilibrium point, then the following simultaneous equations hold.

$$
\begin{gathered}
q_{1}^{*} \lambda_{1} C Q \exp \left\{-q_{1}^{*} \lambda_{1} C\right\} \exp \left\{-C Q q_{2}^{*} \lambda_{2}\right\}=\rho_{2} P_{2} . \\
\exp \left\{-C\left(q_{1}^{*} \lambda_{1}+q_{2}^{*} \lambda_{2} Q\right)\right\}\left(1-q_{1}^{*} \lambda_{1} C\right)=\rho_{1} P_{1} .
\end{gathered}
$$

Solving these simultaneous equation we get the values in (12) and (13). 


\section{APPENDIX B}

PROOF OF LEMMA 1

We calculate the probability that a transmission by a typical node of the Operator is successful in the presence of Jammer.

Proof: Let the independently marked Poisson point process $\tilde{\bar{\Phi}}=\left\{\bar{X}_{i}, \bar{e}_{i}\right\}_{i \geq 1}$ denote the locations and the marks associated with the the transmitters of the Jammer. We assume that $\bar{\Phi}$ is independent of $\tilde{\Phi}$. The marks $\left\{\bar{e}_{i}\right\}_{i \geq 1}$ indicate if a transmitter at location $\bar{X}_{i}$ is turned ON or OFF in a given time slot. A given transmitter is turned $\mathrm{ON}$ with probability $\mathbb{E}\left[\bar{e}_{i}\right]=q_{2}$. Let $\bar{\Phi}^{1}=\left\{\bar{X}_{i}, \bar{e}_{i}=1\right\}$ denote the set of transmitters of the Jammer that are active. Then the shot noise seen by a typical node that is placed at the origin due to the active transmitters of the Jammer is given by $I_{\tilde{\bar{\Phi}}^{1}}=\sum_{\bar{X}_{i} \in \bar{\Phi}^{1}} P_{2} S l\left(\left|\bar{X}_{i}\right|\right)$. For notational simplicity write $I_{1}^{\Phi}=I_{\tilde{\Phi}^{1}}$ and $I_{2}=I_{\tilde{\Phi}^{1}}$. The total interference at the typical node is $I_{1}+I_{2}$. Let $p_{c}\left(q_{1}, q_{2}\right)$ denote the success probability of the typical node when the Operator uses the MAP $q_{1}$ and the Jammer turns ON its transmitters with probability $q_{2}$. Then from the definition of success probability in Equation (2) we have

$$
\begin{aligned}
& p_{c}\left(q_{1}, q_{2}\right) \\
& \quad=\operatorname{Pr}\left\{S l(r) P_{1} \geq T\left(I_{1}+I_{2}+W\right)\right\} \\
& \quad=\mathbb{E}\left\{e^{\frac{-\mu T I_{1}}{l(r) P_{1}}}\right\} \mathbb{E}\left\{e^{\frac{-\mu T I_{2}}{l(r) P_{1}}}\right\} \mathbb{E}\left\{e^{\frac{-\mu T W}{l(r) P_{1}}}\right\} \\
& =\mathbb{E}\left\{e^{\frac{-\mu T W}{l(r) P_{1}}}\right\} \mathcal{L}_{I_{1}}\left(\frac{-\mu T}{l(r) P_{1}}\right) \mathcal{L}_{I_{2}}\left(\frac{-\mu T}{l(r) P_{1}}\right),
\end{aligned}
$$

where Equation (28) follows from the assumptions that the $I_{1}$, $I_{2}$ and $W$ are independent, and $S$ is Rayleigh distributed with mean $1 / \mu$. In Equation (29) $\mathcal{L}_{I_{1}}(s)$ and $\mathcal{L}_{I_{2}}(s)$ denotes the Laplace of shot noise $I_{\tilde{\Phi}^{1}}$ and $I_{\tilde{\Phi}^{1}}$ respectively, evaluated at $s$. We compute each term in the last expression as following:

$$
\begin{aligned}
& \mathcal{L}_{I_{1}}\left(\frac{-\mu T}{l(r) P_{1}}\right) \\
& \quad=\exp \left\{-2 \pi \lambda_{1} q_{1} \int_{u \geq 0}\left(1-\mathbb{E}\left[e^{\frac{-l(u) \mu T}{l(r)}}\right]\right) u \mathrm{~d} u\right\}(30) \\
& \quad=\exp \left\{-2 \pi \lambda_{1} q_{1} \int_{u \geq 0}\left(\frac{u \mathrm{~d} u}{1+l(r) / T l(u)}\right)\right\} \\
& =\exp \left\{-2 \pi \lambda_{1} q_{1} r^{2} T^{2 / \beta} K(\beta)\right\}
\end{aligned}
$$

where Equation (30) follows by applying the definition of Laplace functional of Poisson point process. Equation (31) follows by noting that expectation in Equation (30) is over the Rayleigh distribution. $K(\beta)=\frac{\Gamma(2 / \beta) \Gamma(1-2 / \beta)}{\beta}$. Similarly we calculate

$$
\begin{aligned}
& \mathcal{L}_{I_{2}}\left(\frac{-\mu T}{l(r) P_{1}}\right) \\
& \quad=\exp \left\{-2 \pi \lambda_{2} q_{2} \int_{u \geq 0}\left(1-\mathbb{E}\left[e^{\frac{-P_{2} l(u) \mu T}{l(r) P_{1}}}\right]\right) u \mathrm{~d} u\right\}(33) \\
& \quad=\exp \left\{-2 \pi \lambda_{2} q_{2} \int_{u \geq 0}\left(\frac{u \mathrm{~d} u}{1+P_{1} l(r) / P_{2} T l(r)}\right)\right\} \text { (34) } \\
& \quad=\exp \left\{-2 \pi \lambda_{2} q_{2} r^{2} T^{2 / \beta}\left(P_{2} / P_{1}\right)^{2 / \beta} K(\beta)\right\}
\end{aligned}
$$

Substituting Equations (32), (35) in Equation (29) we get

$p_{c}\left(q_{1}, q_{2}\right)=\mathbb{E}\left\{e^{\frac{-\mu T W}{l(r) P_{1}}}\right\} \exp \left\{-C\left(\lambda_{1} q_{1}+\lambda_{2} q_{2}\left(P_{2} / P_{1}\right)^{2 / \beta}\right)\right\}$.

The intensity of the nodes of the Operator is given by $\lambda_{1} q_{1}$. From the Campbell's formula [2] for the stationary point processes the density of successful transmission is given by multiplying Equation (36) by $\lambda_{1} q_{1}$. This concludes the proof by taking $W \equiv 0$.

\section{APPENDIX C \\ PROOF OF LEMMA 2}

It is easy to observe that the $\bar{U}_{1}\left(q_{1}, \cdot\right)$ is a convex function. Differentiating $\bar{U}_{1}$ with respect to $q_{1}$ once and twice we have

$$
\begin{aligned}
\frac{\partial \bar{U}\left(q_{1}, q_{2}\right)}{\partial q_{1}}=\lambda_{1} & \exp \left\{-C\left(q_{1} \lambda_{1}+q_{2} \lambda_{2} Q\right)\right\} \\
& \times\left(1-q_{1} \lambda_{1} C\right)-\lambda_{1} \rho_{1} P_{1}
\end{aligned}
$$

and

$$
\begin{array}{r}
\frac{\partial^{2} \bar{U}\left(q_{1}, q_{2}\right)}{\partial q_{1}^{2}}=-\lambda_{1} \exp \left\{-C\left(q_{1} \lambda_{1}+q_{2} \lambda_{2} Q\right)\right\} \\
\times\left(2-q_{1} \lambda_{1} C\right)
\end{array}
$$

respectively. First consider the case $\lambda_{1} C \geq 1$. For $q_{1} \geq 1 / \lambda_{1} C$ the slope in (37) is negative. From Equation (38), it is clear that Equation (37) is a decreasing function in $q_{1}$ in the interval $\left[0,1 / \lambda_{1} C\right]$, taking values $\lambda_{1} \exp \left\{-C q_{2} \lambda_{2} Q\right\}-\lambda_{1} \rho_{1} P_{1}>0$ at $q_{1}=0$, and $-\lambda_{1} \rho_{1} P_{1}$ at $q_{1}=1 / \lambda_{1} C$. Thus there exists a unique $q_{1}^{\prime} \in\left[0,1 / \lambda_{1} C\right]$ such that

$$
\exp \left\{-C\left(q_{1}^{\prime} \lambda_{1}+q_{2} \lambda_{2} Q\right)\right\}\left(1-q_{1}^{\prime} \lambda_{1} C\right)=\rho_{1} P_{1} .
$$

For all $q_{1} \leq q_{1}^{\prime}$ the slope of $\bar{U}_{1}\left(\cdot, q_{2}\right)$ is positive and for all $q_{1} \geq q_{1}^{\prime}$ it is negative. Hence we conclude that $\bar{U}_{1}\left(\cdot, q_{2}\right)$ is a quasi concave in $q_{1}$ [13].

For the case $\lambda_{1} C<1$, the second derivative in (38) is negative for all $q_{1} \in[0,1]$. Hence $\bar{U}\left(q_{1}, q_{2}\right)$ is concave in $q_{1}$.

\section{APPENDIX D \\ PROOF OF LEMMA 3}

It is easy to note that $\bar{U}_{1}\left(q_{1}, \cdot\right)$ is concave in $q_{2}$. The concavity in argument $q_{1}$ can be verified by taking the second derivative. The first and second order derivative of $\bar{U}_{1}\left(q_{1},{ }^{\circ}\right)$ with respect to $q_{1}$, after simplification, are as following.

$$
\begin{aligned}
\frac{\partial \bar{U}_{1}\left(q_{1}, q_{2}\right)}{\partial q_{1}}=\frac{\lambda_{1}}{q_{1}^{2}} \exp \left\{C\left(\lambda_{1} q_{1}+\lambda_{2} q_{2} Q\right)\right\} \times \\
\left(1-C \lambda_{1} q_{1}\right)-\lambda_{1} \rho_{1} P_{1}
\end{aligned}
$$


and

$$
\begin{array}{r}
\frac{\partial^{2} \bar{U}_{1}\left(q_{1}, q_{2}\right)}{\partial q_{1}^{2}}=-\frac{\lambda_{1}}{q_{1}} \exp \left\{C\left(\lambda_{1} q_{1}+\lambda_{2} q_{2} Q\right)\right\} \times \\
\left(\left(C \lambda_{1}+1 / q_{1}\right)^{2}+1 / q_{1}\right)
\end{array}
$$

respectively. Equation (40) is negative valued for all $q_{1} \in$ $[0,1]$. Hence $\bar{U}_{1}\left(\cdot, q_{2}\right)$ is a concave function. Now assume that $\lambda_{1} C \geq 1$. As (40) is negative valued, $\partial \bar{U}_{1}\left(q_{1}, q_{2}\right) / \partial q_{1}$ is a decreasing function in $q_{1}$ taking the value $\partial \bar{U}_{1}\left(q_{1}, q_{2}\right) / \partial q_{1}=$ $-\lambda_{1} \rho_{1} P_{1}$ at $q_{1}=1 / \lambda_{1} C$. Hence maximum is achieved in the interval $\left[0,1 / \lambda_{1} C\right]$.

\section{APPENDIX E \\ PROOF OF PROPOSITION 3}

If $\rho_{1} P_{1} \geq 1$ the slope of $\bar{U}$ (INR, $\left.\cdot, q_{2}\right)$ is negative for all values of $q_{2}$. Thus $q_{1}=0$ is the unique maximizer of $\bar{U}\left(\mathrm{INR}, q_{1}, q_{2}\right)$. If $q_{1}=0$ then $q_{2}=0$ is the unique minimizer of $\bar{U}\left(\mathrm{INR}, 0, q_{2}\right)$. Hence $(0,0)$ is an equilibrium. Differentiating anti-potential (19) with respect to $q_{1}$ and $q_{2}$, we have

$$
\begin{aligned}
& \partial \bar{U}\left(\mathrm{INR}, q_{1}, q_{2}\right) / \partial q_{1} \\
& \quad=\frac{\left(C_{1} \lambda_{2} q_{2} Q+\lambda_{0}\right) \lambda_{0}}{\left(C_{1} \lambda_{1} q_{1}+C_{1} \lambda_{2} q_{2} Q+\lambda_{0}\right)^{2}}-\rho_{1} P_{1}
\end{aligned}
$$

and (19) with respect to $q_{1}$

$$
\begin{aligned}
& \partial \bar{U}\left(\mathrm{INR}, \tilde{q}_{1}, q_{2}\right) / \partial q_{2}= \\
& \quad-\quad \frac{\lambda_{1} \lambda_{0} q_{1} C_{1} Q}{\left(C_{1} \lambda_{1} q_{1}+C_{1} \lambda_{2} q_{2} Q+\lambda_{0}\right)^{2}}+\rho_{2} P_{2},
\end{aligned}
$$

respectively. Assume that $\rho_{1} P_{1}<\lambda_{0} /\left(\lambda_{2} C Q+1\right)$. Let $q_{2}=$ 0 , then it follows from Equation (41) that the maximizer of $\bar{U}(\operatorname{INR}, \cdot, 0)$ is $(22)$. Let $q_{1}=\tilde{q}_{1}$, the slope of $\bar{U}\left(\operatorname{INR}, \tilde{q}_{1}, \cdot\right)$ is

which is positive for all values of $q_{2}$ under the hypothesis of the first bullet. This implies that $\bar{U}\left(\mathrm{INR}, \tilde{q}_{1}, \cdot\right)$ is an increasing function in $q_{2}$. Then $q_{2}=0$ is the optimal strategy for the Jammer. This verifies the claim in the first bullet. Assume $q_{2}=1$, it follows from Equation (41) that the maximizer of $\bar{U}$ (INR, $\cdot 1)$ is (23). Let $q_{1}=\bar{q}_{1}$, form Equation (42) the slope of $\bar{U}\left(\mathrm{INR}, \bar{q}_{1}, \cdot\right)$ is negative for all values of $q_{2}$ under the hypothesis of the second bullet. This implies that $\bar{U}\left(\mathrm{INR}, \tilde{q}_{1}, \cdot\right)$ is a decreasing function in $q_{2}$. Then the optimal value for the Jammer is to set $q_{2}=1$. This verifies the claim in second bullet. Let $\left(q_{1}^{*}, q_{2}^{*}\right)$ be an interior equilibrium point, then from (41) and (42) the following simultaneous equations hold.

$$
\begin{gathered}
\frac{\left(C_{1} \lambda_{2} q_{2}^{*} Q+\lambda_{0}\right) \lambda_{0}}{\left(C_{1} \lambda_{1} q_{1}^{*}+C_{1} \lambda_{2} q_{2}^{*} Q+\lambda_{0}\right)^{2}}=\rho_{1} P_{1} \\
\frac{\lambda_{1} \lambda_{0} q_{1}^{*} C_{1} Q}{\left(C_{1} \lambda_{1} q_{1}^{*}+C_{1} \lambda_{2} q_{2}^{*} Q+\lambda_{0}\right)^{2}}=\rho_{2} P_{2}
\end{gathered}
$$

Taking the ratio of the above equations we obtain

$$
\left(C_{1} \lambda_{2} q_{2}^{*} Q+\lambda_{0}\right)=\frac{\rho_{1} P_{1} \lambda_{1} q_{1}^{*} C_{1} Q}{\rho_{2} P_{2}} .
$$

Substituting this relation in (44) we get (24). Using (24) and the relation (44) we obtain (25). 